\title{
Constructing, Analyzing and Synchronizing a Chaotic System with Equilibria of Rectangle Loop
}

\author{
Wei Feng ${ }^{1} 2^{*}$, Yigang $\mathrm{He}^{2}$ \\ ${ }^{1}$ School of Mathematics and Computer Science, Panzhihua University, Panzhihua 617000, China \\ 2 School of Electrical Engineering and Automation, Hefei University of Technology, Hefei 230009, China \\ * Corresponding author. Tel.: 0086 13547613417; email: weifengpzh@sohu.com \\ Manuscript submitted March 28, 2018; accepted May 2, 2018. \\ doi: $10.17706 /$ jsw.13.4.212-218
}

\begin{abstract}
Recent interest has been paid to chaotic systems with infinite number of equilibrium points. In this work, we construct a chaotic system with equilibrium points of rectangle loop. Basic dynamical properties, including phase portrait, equilibrium point and bifurcation of the new system, are investigated numerically and theoretically. Analyses show that this system can generate multiple attractors with different initial values, which reveals its rich dynamics. A simple control scheme with linear controller is designed to synchronize the systems, and the feasibility is verified by the numerical simulations.
\end{abstract}

Key words: linear control, loop equilibrium, multiple attractors, synchronization.

\section{Introduction}

Autonomous systems are at least third-order nonlinear differential equations to behave chaotic with one or more positive Lyapunov exponent. Chaotic system has the properties as sensitive to initial condition, infinite recurrence and bounded orbit. Chaotic behave exists widely in different scientific disciplines including neural network, economy, biology, electrical circuit, etc. [1]-[4]. Since the chaotic model describing the simplified Rayleigh-Benard problem is found by Lorenz [5], there have many interests shown on the theories and applications of chaotic systems.

The equilibrium point of a chaotic system is the real solution of its differential equation, which plays an important role in feature analysis, classification and signal amplitude [6]-[11]. The feature of equilibrium point brings on four types of chaotic systems with heteroclinic orbit, homoclinic orbit, both heteroclinic and homoclinic orbits, without heteroclinic orbit and homoclinic orbit, respectively. Recently, there has been paid growing attention to special chaotic systems with no equilibrium and with uncountable infinite number of equilibrium points [7], [12]-[16]. The finding of these reported systems is striking for their new features of chaos, and leads to a new classification of chaotic attractors as hidden attractor and self-excited attractor from the computational point of view. The attracting basin of hidden attractor does not intersect with any small neighborhoods of its equilibrium point, whereas the self-excited attractor holds a basin of attraction associated with an unstable equilibrium. Therefore, the systems without equilibrium or infinite equilibrium points belong to the type of hidden attractors. However, rare cover seems to be known about the dynamical behavior of the system with infinite equilibrium points.

This paper aims at revealing some mysterious properties of the dynamical system with infinite equilibrium points. We first construct a chaotic system with equilibrium points of rectangle loop. Basic 
dynamical properties including phase portrait, equilibrium point and bifurcation of the new system are investigated numerically and theoretically. And the analyses show that this system holds rich dynamics by generating multiple attractors with different initial values. Then, a simple control scheme with linear controller is designed to synchronize the systems, which is further verified by the numerical simulations.

\section{The Introduced Chaotic System}

In 2015, Gotthans and Petržela reported the new discovery of chaotic dynamics with an infinite number of equilibrium points [14], depicted by the dimensionless set of three first-order differential equations

$$
\left\{\begin{array}{l}
\dot{x}_{1}=a x_{3} \\
\dot{x}_{2}=x_{3} \cdot f_{1}\left(x_{1}, x_{2}, x_{3}\right) \\
\dot{x}_{3}=f_{2}\left(x_{1}, x_{2}, x_{3}\right)+x_{3} \cdot f_{3}\left(x_{1}, x_{2}, x_{3}\right)
\end{array}\right.
$$

where $x_{1}, x_{2}, x_{3}$ are state variables, and $a$ is a free parameter. $f_{1}\left(x_{1}, x_{2}, x_{3}\right), f_{2}\left(x_{1}, x_{2}, x_{3}\right), f_{3}\left(x_{1}, x_{2}, x_{3}\right)$ are three nonlinear functions. The authors of [14] considered the following function form to get a system with circular equilibrium: $f_{1}\left(x_{1}, x_{2}, x_{3}\right)=b x_{1}+c x_{3}^{2}, f_{2}\left(x_{1}, x_{2}, x_{3}\right)=x_{1}{ }^{2}+x_{2}{ }^{2}-r^{2}, f_{3}\left(x_{1}, x_{2}, x_{3}\right)=d x_{1}$. Inspired by Gotthans's work, we proposed a new 3D system with the following form:

$$
\left\{\begin{array}{l}
\dot{x}_{1}=a x_{3} \\
\dot{x}_{2}=x_{3}\left(b x_{1}-c x_{2}-x_{3}^{2}\right) \\
\dot{x}_{3}=x_{1}{ }^{2}+1.5 x_{2}{ }^{4}-r+x_{3}\left(d x_{1}-x_{3}\right)
\end{array}\right.
$$

Taking the condition $\dot{x}_{1}=0, \dot{x}_{2}=0, \dot{x}_{3}=0$, we obtain the rectangle loop equilibrium point of system (2) as $P\left(x_{1 p}, x_{2 p}, 0\right)$, with $x_{1 p}{ }^{2}+1.5 x_{2 p}{ }^{4}-r=0$. The loop shape of equilibrium $P\left(x_{1 p}, x_{2 p}, 0\right)$ for different $r$ is depicted in Fig.1.

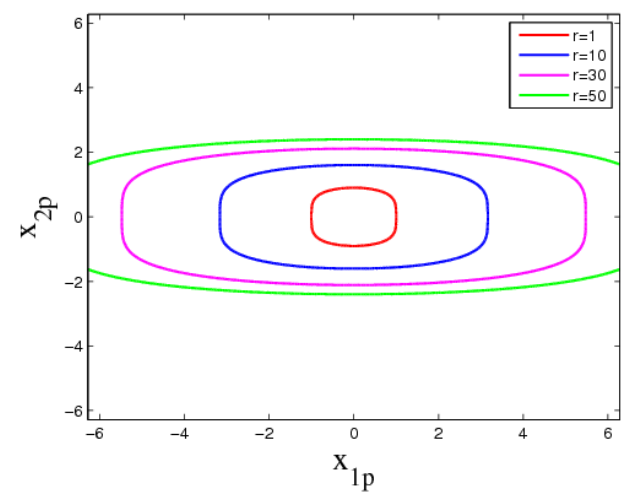

Fig. 1. Shapes of equilibrium points with different $r$.
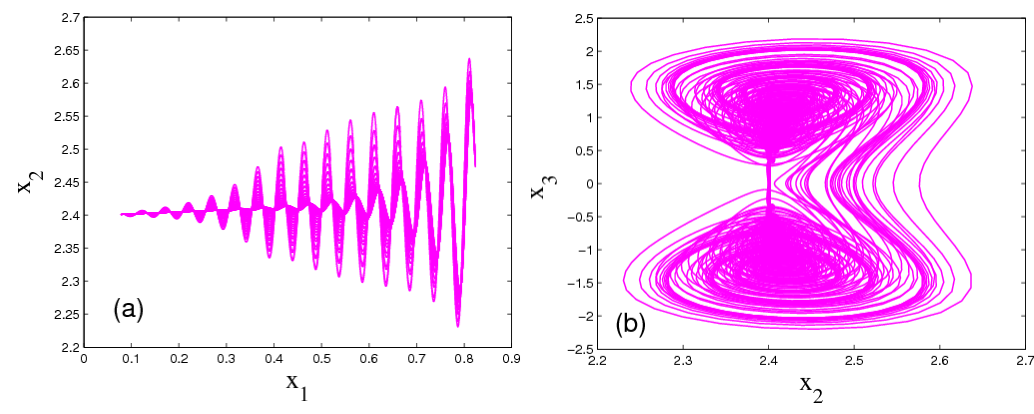

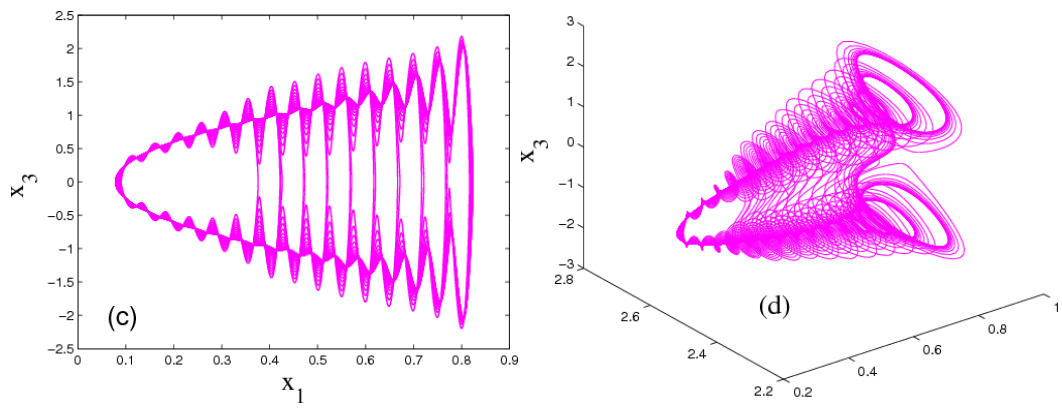

Fig. 2. Chaotic phase diagrams of system (2). (a) $x_{1}-x_{2}$ plane; (b) $x_{2}-x_{3}$ plane; (c) $x_{1}-x_{3}$ plane; (d) $x_{1}-x_{2}-x_{3}$ plane.

When consider $a=-0.1, b=3.0, c=0.1, d=-0.1, r=50$ and initial condition (0.1, 0.6, 0), system (2) exhibits chaotic behavior of multi-layer double-bell attractor, as plotted in Fig.2. The dense dots of Poincaré maps on different crossing planes further verify the emergence of chaos, which is described in Fig.3.
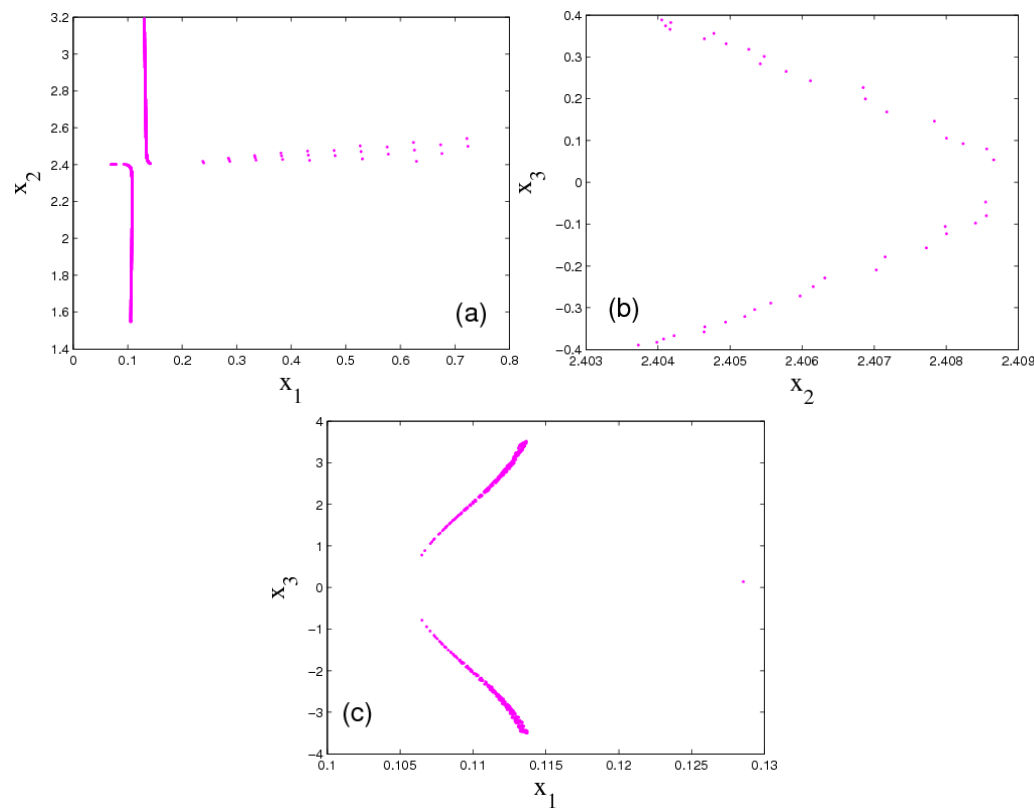

Fig. 3. Poincaré maps on (a) $x_{3}=0$; (b) $x_{1}=0.14$; (c) $x_{2}=1.6$.

\section{Bifurcation Analysis by Varying Parameters}

The reported system has been found to exhibit complex dynamical behaviors by varying system parameters in a wide range. We consider parameter set $a=-0.1, b=3.0, c=0.1, d=-0.1, r=50$ and initial condition $(0.1,0.6,0)$, while let $b$ varies in the region [1], [5] and $r$ varies in the region [0,60], respectively. The corresponding bifurcation diagram and maximal Lyapunov exponent spectrum for system (2) are depicted in Fig.4 and Fig.5. It's known from Fig. 4 that there exists quasi-periodic behavior when $b \in[1,2.1]$, finally falls into chaos when $b>2.1$. But from Fig. 5 we know that there exists a narrow quasi-periodic window when $r \in[0,0.9]$, and system is chaotic when $r>0.9$.

\section{Multiple Attractors with Different Initial Conditions}

One of important feature of the presented system is that it can generate multiple chaotic attractors with different initial condition. To illustrate our finding, we fix $a=-0.1, b=3.0, c=0.1, d=-0.1, r=50$. When consider 
initial condition $x(0)=(0.07,0.6,0)$, system (2) is chaotic; when $x(0)=(0.069,0.6,0)$, system (2) exhibits a periodic orbit; and when we select the initial condition $(0.068,0,0)$, system (2) displays a quasi-periodic behavior. The numerical results are shown in Fig. 6.

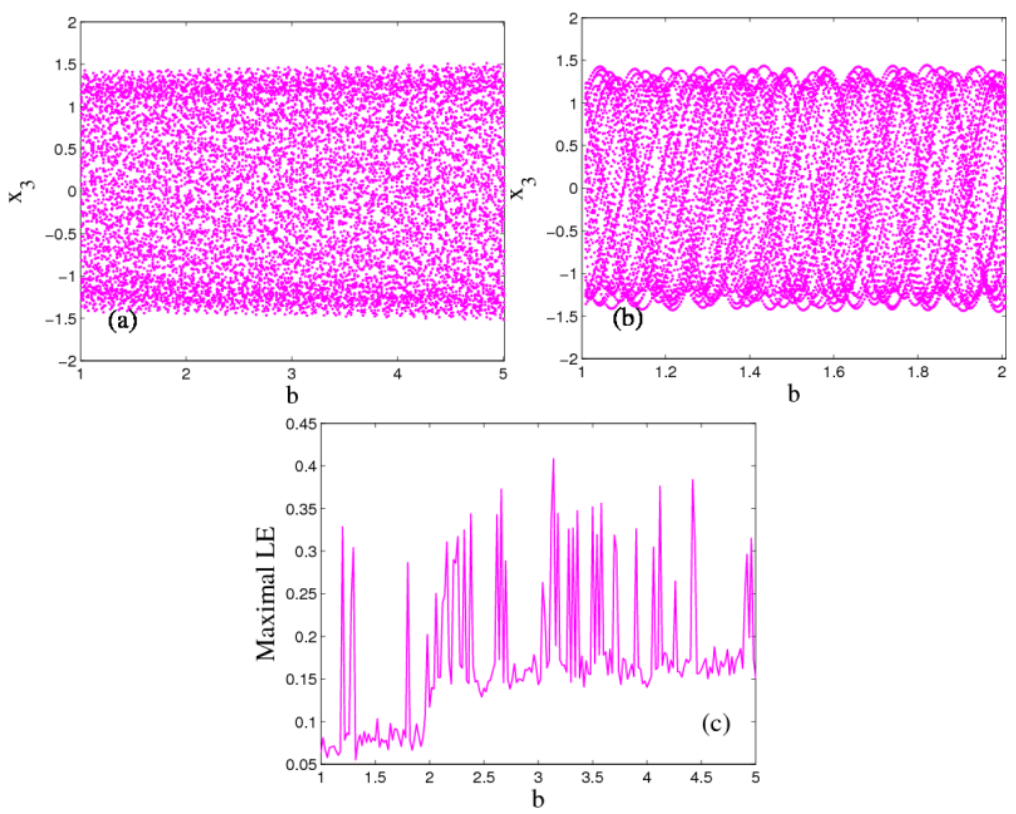

Fig. 4. Bifurcation diagram and maximal Lyapunov exponent spectrum of system (2) versus parameter $b$.
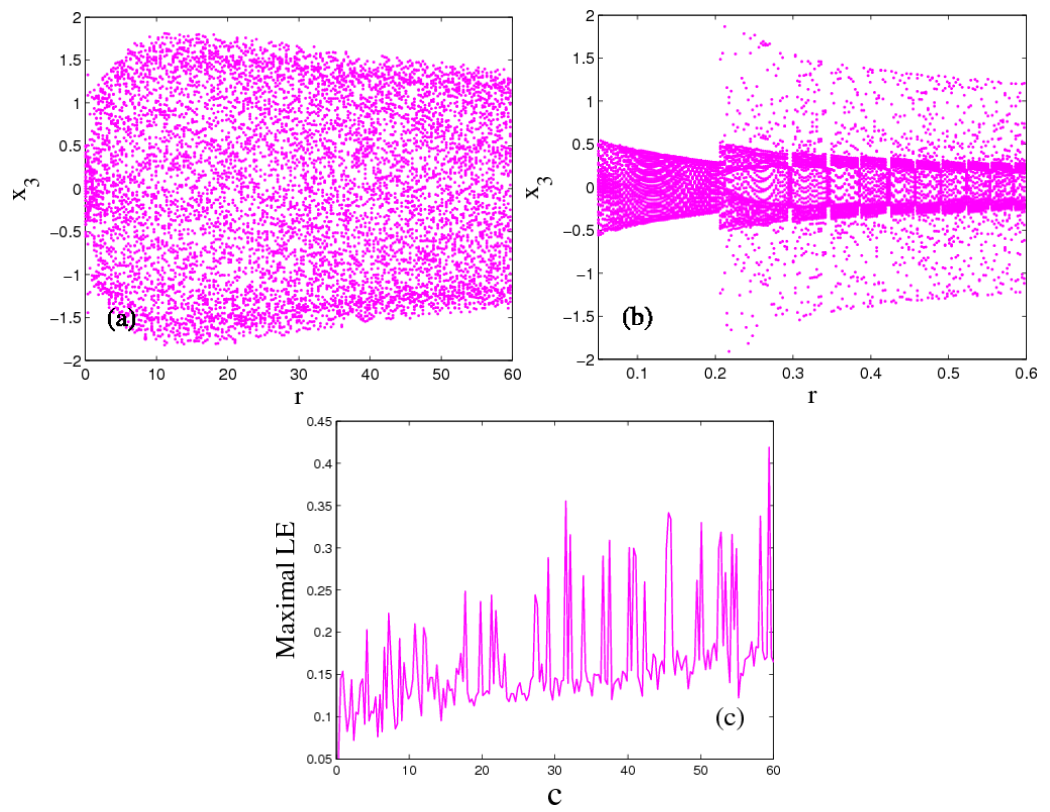

Fig. 5. Bifurcation diagram and maximal Lyapunov exponent spectrum of system (2) versus parameter $r$.

\section{Synchronization of the Introduced Chaotic System}

As we known that two chaotic trajectory will separate exponentially with time starting from slightly different initial conditions. Thereby, chaotic synchronization is deemed to have theoretical and practical applications in secure communication, information science, control processing and biomedical engineering. However, it is impossible for one to synchronize two identical chaotic systems with different initial points, 
even if the exact knowledge of the system model is known. Therefore, a proper synchronization controller is indispensable to achieve synchronization of chaotic systems.

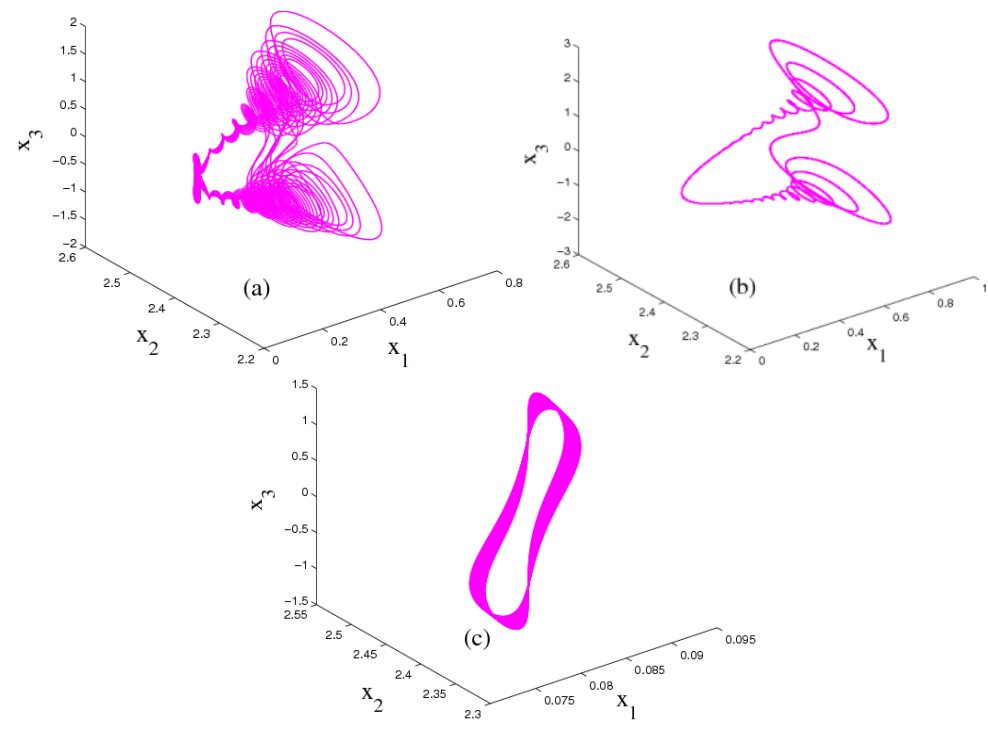

Fig. 6. Multiple attractors of system (2) with initialization as (a) $(0.07,0.6,0)$; (b) $(0.069,0.6,0)$; (c) $(0.068$, $0,0)$.

In this section, a practical control scheme will be designed to synchronize the reported systems. The synchronization scheme is simple with linear controller.

System (2) is taken as the master system, and the controlled slave system is given below

$$
\left\{\begin{array}{l}
\dot{y}_{1}=a y_{3}-k_{1}\left(y_{1}-x_{1}\right) \\
\dot{y}_{2}=y_{3}\left(b y_{1}-c y_{2}-y_{3}^{2}\right)-k_{2}\left(y_{2}-x_{2}\right) \\
\dot{y}_{3}=y_{1}{ }^{2}+1.5 y_{2}{ }^{4}-r+y_{3}\left(d y_{1}-y_{3}\right)
\end{array}\right.
$$

In system (3), $k_{1}, k_{2}$ are the coupled control gains. And we can realize complete synchronization of systems (2) and (3) by taking suitable values of $k_{1}, k_{2}$. We will numerically evaluate the distribution of synchronization region in the controlling-parameter space $\left(k_{1}, k_{2}\right)$. Therefore, we define the synchronization errors as $e_{1}=y_{1}-x_{1}, e_{2}=y_{2}-x_{2}, e_{3}=y_{3}-x_{3}$. And to expediently evaluate the control gains, the minimum synchronization error is considered, described by $E_{\min }=\sqrt{e_{1}^{2}+e_{2}^{2}+e_{3}^{2}}$ in a given finite time $T_{0}$.

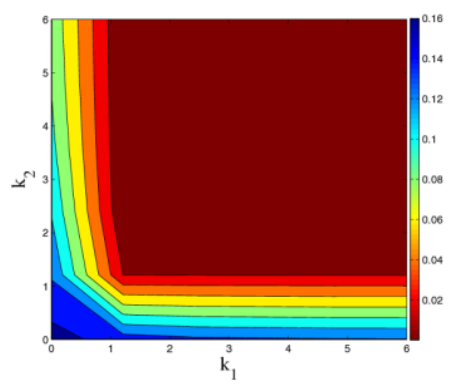

Fig. 7. The distribution of the synchronization error.

In the process of numerical simulation, the system parameter is taken as $a=-0.1, b=3.0, c=0.1, d=-0.1$, $r=50$, and the ODE45 method in Matlab software is employed to solve the differential equations. The initial 
states of systems (2) and (3) are set as $x(0)=(0.1,0.6,0)$, and $y(0)=(0.01,0.2,0.1)$, respectively. When selecting $T_{0}=10$, the distribution of the synchronization error $E_{\min }$ in the controlling-parameter space $\left(k_{1}, k_{2}\right)$ is illustrated in Fig. 7. It's found that the synchronization region appears approximately when $k_{1}>1$ and $k_{2}>1.2$. The simulation result is plotted in Fig. 8 with $k_{1}=2, k_{2}=3$. From the curves of time response and synchronization error of the states in Fig.8, it's known that the chaotic systems (2) and (3) are synchronized.
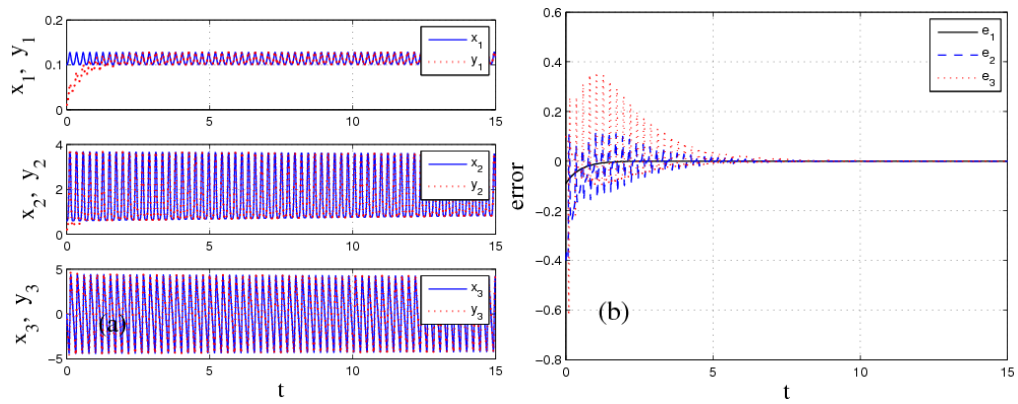

Fig. 8. Synchronisation result: (a) time response of the states; (b) synchronization error.

\section{Conclusion}

In this paper, we mainly aim at revealing some mysterious properties of the dynamical system with infinite equilibrium points. First, we construct a chaotic system with equilibrium points of rectangle loop. Basic dynamical properties including phase portrait, equilibrium point and bifurcation of the new system are investigated numerically and theoretically. And the analyses show that this system holds rich dynamics by generating multiple attractors with different initial values. Then, a simple control scheme with linear controller is designed to synchronize the systems. And by taking suitable coupled control gains, we realized complete synchronization of the reported system numerically, which further verified the theoretical scheme.

\section{Acknowledgment}

This work was supported by the National Natural Science Foundation of China (Grant No. 51577046), the Key Program of the National Natural Science Foundation of China (Grant No. 51637004), the National Key Research and Development Plan "Important Scientific Instruments and Equipment Development" (Grant No.2016YFF0102200) and Central Military Commission Equipment Research Project in Advance (Grant No.41402040301).

\section{References}

[1] Wei, D. Q., Luo, X. S., \& Zhang, B. (2012). Synchronization of brushless DC motors based on LaSalle invariance principle. Nonlinear Dynamics, 69(4), 1733-1738.

[2] Li, C. L., Yang, B. S., Huang, L., Feng, T., He, Y., \& Zou, M. R. (2015). Realization of periodical control and synchronization of single-mode laser Haken-Lorenz system with intermittent feedback. Acta Physica Sinica. 64(3), 30504-030504.

[3] Zhao, Y. B., Zhang, X. Z., Xu, J. \& Guo, Y. C. (2015). Identification of chaotic memristor systems based on piecewise adaptive Legendre filters. Chaos Solitons \& Fractals the Interdisciplinary Journal of Nonlinear Science \& Nonequilibrium \& Complex Phenomena, 81(20), 315-319.

[4] Wei, D. Q., Zhang, B., Luo, X. S., Zeng, S. Y., \& Qiu, D. Y. (2013). Effects of couplings on the collective dynamics of permanent magnet synchronous motors. IEEE Transactions on Circuits \& Systems II Analog \& Digital Signal Processing, 60(10), 692-696.

[5] Lorenz, E. N. (2004). Deterministic nonperiodic flow. Journal of Atmospheric Sciences, 20(2), 130-141. 
[6] Ma, J., Wu, X., Chu, R., \& Zhang. L. (2014). Selection of multi-scroll attractors in Jerk circuits and their verification using Pspice. Nonlinear Dynamics, 76(4), 1-12.

[7] Wei, Z. (2011). Dynamical behaviors of a chaotic system with no equilibria. Physics Letters A, 376(2), 102-108.

[8] Li, C. L., Su, K. L., \& Wu, L. (2013). Adaptive sliding mode control for synchronization of a fractional-order chaotic system. Journal of Computational \& Nonlinear Dynamics, 8(3), 031005-031011.

[9] Li, C. L., Wu, L., Li, H. M., \& Tong, Y. N. (2013). A novel chaotic system and its topological horseshoe. Nonlinear Analysis Modelling \& Control, 1(1), 66-77.

[10] Li, C. L., Su, K. L., \& Zhang, J. (2015). Amplitude control and projective synchronization of a dynamical system with exponential nonlinearity. Applied Mathematical Modelling, 39(18), 5392-5398.

[11] Li, C. L., \& Zhang, J. (2016). Synchronization of a fractional-order chaotic system using finite time input-to-state stability. International Journal of Systems Science, 47(10), 2440-2448.

[12] Sprott, J. C. (2014). A dynamical system with a strange attractor and invariant tori. Physics Letters A, $378(20), 1361-1363$.

[13] Jafari, S., \& Sprott, J. C. (2013). Simple chaotic flows with a line equilibrium. Chaos Solitons \& Fractals the Interdisciplinary Journal of Nonlinear Science \& Nonequilibrium \& Complex Phenomena, 57(4), 79-84.

[14] Gotthans, T., \& Petržela, J. (2015). New class of chaotic systems with circular equilibrium. Nonlinear Dynamics, 81(3), 1-7.

[15] Kingni, S. T., Pham, V. T., Jafari, S., Kol, G. R., \& Woafo, P. (2016). Three-dimensional chaotic autonomous system with a circular equilibrium: Analysis, circuit implementation and its fractional-order form. Circuits Systems \& Signal Processing, 35(6), 1933-1948.

[16] Chen, Y. M. \& Yang, Q. G. (2015). A new Lorenz-type hyperchaotic system with a curve of equilibria. Mathematics \& Computers in Simulation, 112, 40-55.

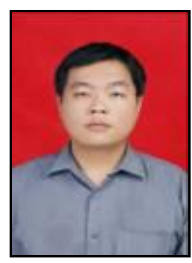

Wei Feng received M.Sc degree in computer applied technology from Chongqing University of Posts and Telecommunications of China in 2007. He is a Ph.D. candidate in the Hefei University of Technology. He is also a lecturer at Panzhihua University. His general research interests include chaos system and chaotic encryption.

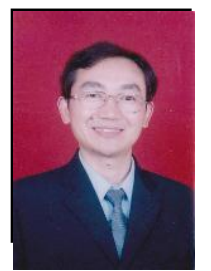

Yigang He received the M.Sc. degree in electrical engineering from Hunan University, Changsha, China, in 1992 and the Ph.D. degree in electrical engineering from Xi'an Jiaotong University, Xi'an,China, in 1996. In 1990, he joined the College of Electrical and Information Engineering, Hunan University and was promoted to Associate Professor, Professor in 1996, 1999, respectively. From 2006 to 2011, he worked as the Director of the Institute of Testing Technology for Circuits and Systems, Hunan University. He was a Senior Visiting Scholar with the University of Hertfordshire, Hatfield, U.K., in 2002. In 2011, he joined the Hefei University of Technology, China, and currently works as the Head of School of Electrical Engineering and Automation, Hefei University of Technology. His teaching and research interests are in the areas of circuit theory and its applications, testing and fault diagnosis of analog and mixed-signal circuits, electrical signal detection, smart grid, radio frequency identification technology, and intelligent signal processing. He has published some 200 journal and conference papers in the aforementioned areas and several chapters in edited books. Dr. He has been on the Technical Program Committees of a number of international conferences. He was the recipient of a number of national and international awards, prizes, and honors 